\title{
Some explicit constructions of sets with more sums than differences
}

\author{
by \\ Peter V. Hegarty (Göteborg)
}

1. Introduction. If $A \subseteq \mathbb{Z}$ the sumset and difference set of $A$ are defined, respectively, as

$$
\begin{aligned}
& A+A:=\left\{x \in \mathbb{Z}: x=a_{1}+a_{2} \text { for some } a_{1}, a_{2} \in A\right\}, \\
& A-A:=\left\{x \in \mathbb{Z}: x=a_{1}-a_{2} \text { for some } a_{1}, a_{2} \in A\right\} .
\end{aligned}
$$

If $A$ is a finite set with $n$ elements then it is easy to see that, a priori,

$$
\begin{aligned}
& 2 n-1 \leq|A+A| \leq n(n+1) / 2, \\
& 2 n-1 \leq|A-A| \leq n(n-1)+1 .
\end{aligned}
$$

The upper bounds follow simply from the fact that addition is commutative whereas subtraction is not, and this certainly suggests that "most" (speaking informally) finite sets $A$ should have the property that $|A+A| \leq|A-A|$. A precise result in support of this intuition was proven by Roesler [6]: he showed that for any $n>0$ and $1 \leq k \leq n$, the average value of the quotient $|A-A| /|A+A|$, as $A$ runs over the $k$-element subsets of $\{0,1, \ldots, n\}$, lies in the interval $[1,2)$. A question of murky origins, but dating back to the $1960 \mathrm{~s}$, is whether there exist any finite sets $A$ of integers at all such that $|A+A|>$ $|A-A|$. The question is interesting because of the apparent difficulty in finding examples of such sets, whereas it is very easy to construct sets $A$ with $|A+A|=|A-A|$. Any arithmetic progression has this property or, more generally, any set $A$ with the property that $A=\{x\}-A$ for some $x \in \mathbb{Z}$. Such a set is said to be symmetric (about $x / 2$ ).

Following the practice introduced by Nathanson [4], we refer to sets having more sums than differences as MSTD sets. Some examples of such sets appear in the literature from the late 1960s and early 1970s. Conway is said to have found the example $A_{1}=\{0,2,3,4,7,11,12,14\}$. The example $A_{2}=\{0,1,2,4,7,8,12,14,15\}$ appears in [2]. Another interest-

2000 Mathematics Subject Classification: Primary 11B75; Secondary 11K99.

Key words and phrases: sumsets, difference sets, MSTD sets. 
ing example, $A_{3}=\{0,1,2,4,5,9,12,13,14,16,17,21,24,25,26,28,29\}$, appears in [1]. Note that for $i=1,2,\left|A_{i}+A_{i}\right|-\left|A_{i}-A_{i}\right|=1$, whereas $\left|A_{3}+A_{3}\right|-\left|A_{3}-A_{3}\right|=4$.

The following two observations are pertinent:

1. The property of being an MSTD set is invariant under linear mappings $x \mapsto u x+v, u, v \in \mathbb{Z}, u \neq 0$. The sizes of sum- and difference sets are unchanged by such mappings. In particular, it suffices to consider finite subsets of $\mathbb{Z}$ which have smallest element 0 , and such that the greatest common divisor of the elements in the set is 1 . Such sets will be called normalised.

2. Starting from any MSTD set $A$ we can construct a sequence $A=$ $A_{1}, A_{2}, \ldots$ of MSTD sets such that the quotients $\left|A_{t}+A_{t}\right| /\left|A_{t}-A_{t}\right|$ become arbitrarily large. Choose an integer $m$ and set

$$
A_{t}:=\left\{\sum_{i=1}^{t} a_{i} m^{i-1}: a_{i} \in A\right\} .
$$

If $m$ is sufficiently large, then $\left|A_{t} \pm A_{t}\right|=|A \pm A|^{t}$. This method of constructing an infinite family of MSTD sets from a single one will be called the base expansion method.

In particular, these observations imply that any MSTD set gives rise to an infinite family of such sets. It appears to have been an open problem for some time to find some other way of constructing an infinite family of MSTD sets. In [10], Ruzsa uses probabilistic arguments to prove the existence of a multitude of MSTD sets. However, this still does not provide explicit constructions. Extending an observation of the author and Roesler, such constructions were eventually provided by Nathanson [4]. The idea for his type of construction comes from examples like $A_{1}$ above. Note that that set is the union of a symmetric set $\{0,2,3,7,11,12,14\}$ and the single number 4 . The symmetric part contains an arithmetic progression $\{3,7,11\}$, with some extra numbers tagged on at both ends. Nathanson's sets have this type of structure. One starts with a (proper generalised) arithmetic progression, adds on some structure at both ends while retaining symmetry, then adds one further element which results in the sumset being enlarged by one element while the difference set is left unchanged.

Nathanson's paper also uses a probabilistic method (inspired by Tao) to prove the existence of many MSTD sets in finite abelian groups of the form $\mathbb{Z} / n \mathbb{Z} \times \mathbb{Z} / 2 \mathbb{Z}$, and presents a general method for transforming MSTD sets in finite abelian groups to MSTD sets in $\mathbb{Z}$. Even more recently, Martin and O'Bryant [3] also use probabilistic methods to prove the following impressive result: there is a positive constant $c$ such that, for all $n \gg 0$, at least $c \cdot 2^{n+1}$ subsets of $\{0,1,2, \ldots, n\}$ are MSTD sets. 
In summary, probabilistic methods have shown that the phenomenon of MSTD sets is actually quite common. But explicit constructions of such sets remain hard to come by, with Nathanson's idea being essentially the only one in print. This issue of explicitly constructing MSTD sets will be our primary concern here. We were motivated by one of the questions Nathanson posed in his talk [5], namely whether there existed any MSTD sets in $\mathbb{Z}$ of smaller cardinality than $A_{1}$ above. Our first result is

ThEOREM 1. There are no MSTD subsets of $\mathbb{Z}$ of size 7. Moreover, up to linear transformations, $A_{1}$ is the unique such set of size 8.

Clearly, the classification of all MSTD sets of a given size, up to linear transformation, is a finite computation. To reduce the complexity of the computation to something manageable, even for very small sizes, is quite another matter. Our method accomplishes this for sets of size 8, but even for size 9, the computation was not feasible. We did manage to find all MSTD sets $A$ of size 9 with the following property: for some element $x$ of the sumset $A+A$ there are at least four ordered pairs $\left(a_{1}, a_{2}\right) \in A \times A$ with $a_{1}+a_{2}=x$. There are exactly nine such sets up to linear transformation, namely

$$
\begin{aligned}
A_{4} & =\{0,1,2,4,5,9,12,13,14\}, \\
A_{2} & =\{0,1,2,4,7,8,12,14,15\}, \\
A_{5} & =\{0,2,3,4,7,9,13,14,16\}, \\
A_{6} & =\{0,2,3,4,7,11,12,14,16\}, \\
A_{7} & =\{0,2,3,4,7,11,15,16,18\}, \\
A_{8} & =\{0,2,4,8,9,10,15,17,19\}, \\
A_{9} & =\{0,4,6,7,8,14,15,17,21\}, \\
A_{10} & =\{0,5,6,9,10,13,16,17,22\}, \\
A_{11} & =\{0,4,6,8,11,14,19,21,25\} .
\end{aligned}
$$

Note that $A_{2}$ is the same set as written earlier with the same name. $A_{4}$ is a subset of $A_{3}$. The sets $A_{4}, A_{5}, A_{7}$ appear in [4]. Of these, $A_{4}$ and $A_{7}$ are, along with $A_{1}$, among the infinite family of MSTD sets constructed in Theorem 1 of that paper, whereas $A_{5}$ is among the family of MSTD sets described in Theorem 2 there. The remaining sets $A_{6}, A_{8}, A_{9}, A_{10}, A_{11}$ appear to be new. Working from these examples we will present four constructions of infinite families of MSTD sets (Theorems $2 / 3,4,5,6$ below) which generalise respectively $A_{11} / A_{9}, A_{8}, A_{6}$ and $A_{4}$. All constructions share common features with, but are nevertheless different from in a non-trivial sense, those in [4] and one another. This reflects the main theme of the paper, namely that while the most easily describable MSTD sets all seem to have a com- 
mon core of features, within this framework there is substantial room for variety. The last part of the paper deals with the question

"How much larger can the sumset be than the difference set?"

On the one hand, we will answer a question of [3] (a weaker version was posed in [4]) by showing (Theorem 8) that for every pair $j, k$ of non-negative integers there is a positive constant $c_{j, k}$ such that, for all $n \gg_{j, k} 0$, at least $c_{j, k} \cdot 2^{n+1}$ of the subsets $A$ of $\{0,1, \ldots, n\}$ satisfy $|A+A|=(2 n+1)-j$, $|A-A|=(2 n+1)-2 k$. The proof, which also provides explicit examples for every $j$ and $k$, involves two ideas: firstly, extending the probabilistic method of [3], and secondly relating MSTD sets in $\mathbb{Z}$ to MSTD sets in suitably chosen finite cyclic groups.

On the other hand, the base expansion method suggests that the right quantity to look at when studying the above question is not $|A+A|-|A-A|$ but rather

$$
f(A):=\frac{\ln |A+A|}{\ln |A-A|} .
$$

Results due to Freiman-Pigarev [1] and Ruzsa [7-9] establish that

$$
3 / 4 \leq f(A) \leq 4 / 3
$$

for any finite set $A \subseteq \mathbb{Z}$. It is not known if either bound is sharp, and the state of knowledge is far worse for the upper bound. Ruzsa's probabilistic method [10] produces a constant $c>1$ such that there are a "multitude" of sets $A$ with $f(A)>c$. He does not compute $c$ explicitly, but a quick analysis of his method shows that it gives $c \approx 1+10^{-9}$. The set $A_{3}$ above satisfies $f\left(A_{3}\right)=\ln 59 / \ln 55=1.0175 \ldots$ and there appears to be nothing in print which beats this. We will give explicit examples of sets which do so, if only slightly.

The remainder of the paper is organised as follows. In Section 2 we will prove Theorem 1. A Mathematica code was written to perform the computations necessary to complete the proof. In Section 3 we will first indicate the computations performed which allowed us to conclude that the list of sets $A_{2}, \ldots, A_{11}$ was complete in the sense mentioned above. Then we will prove Theorems $2-6$. In Section 4 we will prove Theorem 8 and exhibit the sets $A$ with larger values of $f(A)$ than anything previously written down. In Section 5 , we will give some concluding remarks and suggestions for further investigations.

2. Proof of Theorem 1. Clearly, it suffices to prove the second statement of the theorem. The location of all MSTD subsets of $\mathbb{Z}$ of a certain size can be represented as a finite computation as follows: 
Let $A$ be a set of size $n>0$, with $A=\left\{a_{i}: i=1, \ldots, n\right\}$ and $a_{1}>\cdots>a_{n}=0$. For $i=1, \ldots, n-1$, represent the difference $a_{i}-a_{i+1}$ as $\vec{e}_{i}$, the $i$ th standard basis vector $\left({ }^{1}\right)$ in $\mathbb{R}^{n-1}$. A computer program, if now asked to compute those quantities, will return $|A+A|=n(n+1) / 2$, $|A-A|=n(n-1)+1$. So if $A$ is to be an MSTD set, there must be a non-trivial coincidence of differences. That is, there must exist $i, j, k, l$ such that $a_{i}-a_{j}=a_{k}-a_{l}$ and $(i, j) \neq(k, l)$. Given such an equation we can, by projection onto the orthogonal complement in $\mathbb{R}^{n-1}$ of the subspace spanned by $\left(\vec{e}_{i}-\vec{e}_{j}\right)-\left(\vec{e}_{k}-\vec{e}_{l}\right)$, represent the elements of $A$ by vectors in $\mathbb{R}^{n-2}$ and recompute $|A+A|$ and $|A-A|$. If still $|A+A| \leq|A-A|$ we can pick another non-trivial identification of elements in $A-A$, and repeat the above procedure, with the elements of $A$ now represented by vectors in $\mathbb{R}^{n-3}$. Clearly, the computation will terminate with all MSTD sets of size $n$ and smallest element zero located, possibly including infinite parameterised families of such sets.

However, the above computation seems to be practically rather unfeasible even for $n=8$. We estimate that our machine would have taken several weeks at least to finish the calculation. For $n=8$ one starts with vectors in $\mathbb{R}^{7}$. It turns out nevertheless that, with a modest amount of argument, one can show that if $A$ is an MSTD set, then there must appear one of 18 possible configurations, each of which reduces the problem to $\mathbb{R}^{4}$ or $\mathbb{R}^{5}$. We thus allowed our program to instead examine each of these 18 possibilities in turn, and the average running time was about 45 minutes. Though the argument used to simplify matters could be pushed further, it was not obvious to us how to do so without an effort which would essentially balance out the resulting reduction in computing time. Thus our proof of Theorem 1 will consist of two parts:

- Part 1: reduction to 18 possible cases as described above.

- Part 2: a computer program to search through all these cases in turn.

PART 1. As before, let $A \subset \mathbb{Z}$ be a set of size $8, A=\left\{a_{i}: i=1, \ldots, 8\right\}$ where $a_{1}>\cdots>a_{8}=0$. We introduce some further notation. Let

$$
\begin{array}{ll}
S:=\left\{\left(a_{i}, a_{j}\right): 1 \leq i \leq j \leq 8\right\}, & \mathcal{S}:=A+A=\left\{a_{i}+a_{j}:\left(a_{i}, a_{j}\right) \in S\right\}, \\
D:=\left\{\left(a_{i}, a_{j}\right): 1 \leq i<j \leq 8\right\}, & \mathcal{D}:=(A-A)_{>0}=\left\{a_{i}-a_{j}:\left(a_{i}, a_{j}\right) \in D\right\} .
\end{array}
$$

Let $\mathcal{R}_{1}$ and $\mathcal{R}_{2}$ be the equivalence relations on $S$ and $D$ respectively defined by

$$
\begin{aligned}
& \left\{\left(a_{i}, a_{j}\right),\left(a_{k}, a_{l}\right)\right\} \in \mathcal{R}_{1} \Leftrightarrow a_{i}+a_{j}=a_{k}+a_{l}, \\
& \left\{\left(a_{i}, a_{j}\right),\left(a_{k}, a_{l}\right)\right\} \in \mathcal{R}_{2} \Leftrightarrow a_{i}-a_{j}=a_{k}-a_{l} .
\end{aligned}
$$

$\left({ }^{1}\right)$ Actually, in our computer program we represent $a_{i}-a_{i+1}$ initially by $\vec{e}_{n-i}$. 
Let $\Sigma:=S / \mathcal{R}_{1}$ and $\Delta:=D / \mathcal{R}_{2}$. Obviously one can identify $\Sigma$ with $\mathcal{S}$ and $\Delta$ with $\mathcal{D}$, but it will be convenient for us to have a separate notation when referring to the equivalence relations. Let $|\Sigma|:=\sigma$ and $|\Delta|:=\delta$. Thus $|A+A|=\sigma$ and $|A-A|=2 \delta+1$. So if $A$ is an MSTD set then

$$
2 \delta+1<\sigma .
$$

Definition. An equivalence class in $\Sigma$ will be said to be nice if it contains at least three elements $\left(a_{i}, a_{j}\right),\left(a_{k}, a_{l}\right),\left(a_{m}, a_{n}\right)$ such that all six indices $i, j, k, l, m, n$ are distinct.

One readily checks that if there is a nice $\Sigma$-class, then $A$ must contain, up to symmetry, one of the following 16 configurations:

$$
\begin{aligned}
& a_{1}+a_{8}=a_{2}+a_{7}=a_{3}+a_{6}, \\
& a_{1}+a_{8}=a_{2}+a_{7}=a_{3}+a_{5}, \\
& a_{1}+a_{8}=a_{2}+a_{7}=a_{3}+a_{4}, \\
& a_{1}+a_{8}=a_{2}+a_{7}=a_{4}+a_{5}, \\
& a_{1}+a_{8}=a_{2}+a_{6}=a_{3}+a_{5}, \\
& a_{1}+a_{8}=a_{2}+a_{6}=a_{3}+a_{4}, \\
& a_{1}+a_{8}=a_{2}+a_{6}=a_{4}+a_{5}, \\
& a_{1}+a_{8}=a_{2}+a_{5}=a_{3}+a_{4}, \\
& a_{1}+a_{8}=a_{3}+a_{6}=a_{4}+a_{5}, \\
& a_{1}+a_{7}=a_{2}+a_{6}=a_{3}+a_{5}, \\
& a_{1}+a_{7}=a_{2}+a_{6}=a_{3}+a_{4}, \\
& a_{1}+a_{7}=a_{2}+a_{6}=a_{4}+a_{5}, \\
& a_{1}+a_{7}=a_{2}+a_{5}=a_{3}+a_{4}, \\
& a_{1}+a_{7}=a_{3}+a_{6}=a_{4}+a_{5}, \\
& a_{1}+a_{6}=a_{2}+a_{5}=a_{3}+a_{4}, \\
& a_{2}+a_{7}=a_{3}+a_{6}=a_{4}+a_{5} .
\end{aligned}
$$

Let $n_{1} \leq \cdots \leq n_{\delta}$ be the sizes of the $\Delta$-classes, arranged in some increasing order. Hence

$$
\sum_{i=1}^{\delta} n_{i}=|D|=28 .
$$

Note that $n_{1}=1$ since $\left(a_{1}, a_{8}\right)$ is in a class by itself. If $\left(a_{i}, a_{j}\right)$ is $\mathcal{R}_{2^{-}}$ equivalent to $\left(a_{k}, a_{l}\right)$, where $i \leq k \leq j \leq l$, then $\left(a_{i}, a_{l}\right)$ is $\mathcal{R}_{1}$-equivalent to $\left(a_{k}, a_{j}\right)$. This defines a mapping $\phi$ from $\mathcal{R}_{2}$-equivalent pairs of elements of $D$ to $\mathcal{R}_{1}$-equivalent pairs of elements of $S$. The mapping is obviously at most $2-1$, hence its range consists of at least $\frac{1}{2} \sum_{i=1}^{\delta}\left(\begin{array}{c}n_{i} \\ 2\end{array}\right)$ pairs. If, in 
addition, there are no nice $\Sigma$-classes, it follows that

$$
\sigma \leq|S|-\frac{1}{2} \sum_{i=1}^{\delta}\left(\begin{array}{c}
n_{i} \\
2
\end{array}\right)=36-\frac{1}{2} \sum_{i=1}^{\delta}\left(\begin{array}{c}
n_{i} \\
2
\end{array}\right) .
$$

We examine the following two cases:

- Case I: $n_{2}>1$.

- Case II: $n_{2}=1$ and there are no nice $\Sigma$-classes.

First consider Case I. Then every $\Delta$-class, other than that consisting of the single pair $\left(a_{1}, a_{8}\right)$, contains at least two members of $D$. First of all this forces $a_{1}-a_{7}=a_{2}-a_{8}$, hence

$$
a_{1}+a_{8}=a_{2}+a_{7} .
$$

Next consider the three differences $a_{1}-a_{6}, a_{2}-a_{7}$ and $a_{3}-a_{8}$. The two largest ones must be equal. If $a_{1}-a_{6}=a_{3}-a_{8}$ then $a_{1}+a_{8}=a_{3}+a_{6}$, which, together with (21), implies that $A$ contains the nice configuration (3). Otherwise, up to symmetry, we may assume that $a_{1}-a_{6}=a_{2}-a_{7}>a_{3}-a_{8}$. It is then easily checked that one of the following three possibilities must hold:

(a) $a_{1}-a_{5}=a_{3}-a_{8}$. This, together with (21), implies that $A$ contains the nice configuration (4).

(b) $a_{1}-a_{5}=a_{2}-a_{6}$. Thus in $A$ we have

$$
a_{1}-a_{2}=a_{5}-a_{6}=a_{6}-a_{7}=a_{7}-a_{8} .
$$

(c) $a_{2}-a_{6}=a_{3}-a_{8}$. Thus in $A$ we have

$$
a_{1}-a_{2}=a_{6}-a_{7}=a_{7}-a_{8}=\frac{1}{2}\left(a_{2}-a_{3}\right) .
$$

This completes the analysis of Case I. We have shown that, under these circumstances, either there is a nice $\Sigma$-class or, up to symmetry, $A$ contains one of the configurations (22) and (23).

Next consider Case II. We claim that in this case, $A$ cannot be an MSTD set. Suppose otherwise. A priori, $\sigma \leq 36$ so (2) forces $\delta \leq 17$. Now this plus (19) and the assumption that $n_{2}=1$ mean that $\sum_{i=1}^{\delta}\left(\begin{array}{c}n_{i} \\ 2\end{array}\right) \geq 11$. Then (20) implies that $\sigma \leq 30$. The idea is now to iterate this kind of argument to gradually reduce $\delta$ until we obtain the contradiction that $\delta<7$.

- If $\sigma \leq 30$ then (2) forces $\delta \leq 14$. Then (19) and the assumption that $n_{2}=1$ force $\sum_{i=1}^{\delta}\left(\begin{array}{c}n_{i} \\ 2\end{array}\right) \geq 16$. Thus (20) forces $\sigma \leq 28$.

- If $\sigma \leq 28$ then (2) forces $\delta \leq 13$. Then (19) and the assumption that $n_{2}=1$ force $\sum_{i=1}^{\delta}\left(\begin{array}{c}n_{i} \\ 2\end{array}\right) \geq 19$. Thus (20) forces $\sigma \leq 26$. 
- If $\sigma \leq 26$ then (2) forces $\delta \leq 12$. Then (19) and the assumption that $n_{2}=1$ force $\sum_{i=1}^{\delta}\left(\begin{array}{c}n_{i} \\ 2\end{array}\right) \geq 22$. Thus (20) forces $\sigma \leq 25$.

- If $\sigma \leq 25$ then (2) forces $\delta \leq 11$. Then (19) and the assumption that $n_{2}=1$ force $\sum_{i=1}^{\delta}\left(\begin{array}{c}n_{i} \\ 2\end{array}\right) \geq 25$. Thus (20) forces $\sigma \leq 23$.

- If $\sigma \leq 23$ then (2) forces $\delta \leq 10$. Then (19) and the assumption that $n_{2}=1$ force $\sum_{i=1}^{\delta}\left(\begin{array}{c}n_{i} \\ 2\end{array}\right) \geq 30$. Thus (20) forces $\sigma \leq 21$.

- If $\sigma \leq 21$ then (2) forces $\delta \leq 9$. Then (19) and the assumption that $n_{2}=1$ force $\sum_{i=1}^{\delta}\left(\begin{array}{c}n_{i} \\ 2\end{array}\right) \geq 36$. Thus (20) forces $\sigma \leq 18$.

- If $\sigma \leq 18$ then (2) forces $\delta \leq 8$. Then (19) and the assumption that $n_{2}=1$ force $\sum_{i=1}^{\delta}\left(\begin{array}{c}n_{i} \\ 2\end{array}\right) \geq 44$. Thus (20) forces $\sigma \leq 14$.

- If $\sigma \leq 14$ then (2) forces $\delta \leq 6$ and we have our desired contradiction.

This completes the analysis of Case II. We have thus shown that if $A$ is an MSTD set then, up to symmetry, either it contains one of the 16 nice configurations (3)-(18), or one of the configurations (22) and (23). Thus the proof of Theorem 1 is reduced to 18 possible cases, as claimed, and we have completed Part 1 of the proof.

PART 2. If $A$ contains a nice configuration, then the differences $a_{i}-a_{i+1}$ can be represented with vectors in $\mathbb{R}^{5}$. If either (22) or (23) hold, then $\mathbb{R}^{4}$ suffices. We can then write a program to search for normalised MSTD sets as outlined above. The rather unwieldy Mathematica code for such a program, with each of the 18 possible input configurations, can be obtained from the author. The total running time for the program on our network was about 15 hours. The only output MSTD sets were $A_{1}$ and $14-A_{1}$, thus proving Theorem 1.

3. Explicit families of MSTD sets. If $A$ is an MSTD set of size 9, then the same type of reasoning as above can be used to show that either there is a nice $\Sigma$-class or $A$ contains one of a small number of other possible configurations analogous to (22) and (23). However, a nice $\Sigma$-class only allows us now to represent the differences $a_{i}-a_{i+1}$, a priori, as vectors in $\mathbb{R}^{6}$. Plus, the number of possible configurations is now much larger. The resulting computation was not practically feasible with our code, so we have not obtained with certainty a full classification of all MSTD sets of size 9, up to linear transformations. Instead, we classified all such sets in which some $\Sigma$-class contains at least four elements. One readily verifies that this leaves, up to symmetry, 25 possible configurations in $A$ analogous to those in (3)-(18). The computer then located, over a period of about two weeks and after multiple crashes, the nine normalised MSTD sets $A_{2}, A_{4}, A_{5}, \ldots$ listed in the introduction. We now present a sequence of constructions of infinite families of MSTD sets which include five of these nine examples: 
Theorem 2. Let $n \geq 3$ and $0 \leq k \leq n-2$. Let $d>1$ be a divisor of $2^{n-k}-1$. Set

$$
\begin{gathered}
X:=\left\{2^{n}-2^{j}: k \leq j \leq n\right\}, \quad m:=\left(2^{n+1}+d\right)+\left(2^{n}-2^{k}\right), \quad Y:=m-X, \\
Z:=\left\{2^{n}+j d: 1 \leq j \leq \frac{2^{n}-2^{k}}{d}\right\}, \\
B:=X \sqcup Y \sqcup Z, \quad a:=2^{n}, \quad A:=B \cup\{a\} .
\end{gathered}
$$

Then $A$ is a normalised MSTD set with $|A+A|=|A-A|+1$.

Proof. One may verify the following facts:

(i) $B$ is a symmetric set, $B=m-B$.

(ii) $A-A=B-B$.

(iii) $A+A=(B+B) \sqcup\{2 a\}$.

The verifications involve calculations similar to those appearing in the proofs of Theorems 1-4 in [4], hence are omitted.

Remark. The set $A_{11}$ is the case $n=3, k=1, d=3$ of the above theorem.

In the notation of Theorem 2 , when $k=0$ there is a simpler, similar construction of a family of normalised MSTD sets; basically the arithmetic progression $Z$ is not needed.

Theorem 3. Let $n \geq 3$ and $1 \leq l \leq n-2$. Set

$$
\begin{gathered}
X:=\left\{2^{n}-2^{j}: 0 \leq j \leq n\right\}, \quad m:=\left(2^{n+1}-1\right)+\left(2^{n}-2^{l}\right), \quad Y:=m-X, \\
B:=X \sqcup Y, \quad a:=2^{n}, \quad A:=B \sqcup\{a\} .
\end{gathered}
$$

Then $A$ is a normalised MSTD set with $|A+A|=|A-A|+1$.

Proof. As for Theorem 2. Observe that $A_{9}$ is the case $n=3, l=1$ of the theorem.

Next we turn to a generalisation of $A_{8}$ :

Theorem 4. Let $n, d>1$. Set

$$
\begin{gathered}
X:=\{j d: 0 \leq j \leq n\}, \quad m:=(4 n+1) d+1, \quad Y:=m-X, \\
Z:=(2 n d,(2 n+1) d], \quad B:=X \sqcup Y \sqcup Z, \quad a:=2 n d, \quad A:=B \sqcup\{a\} .
\end{gathered}
$$

Then $A$ is a normalised $M S T D$ set with $|A+A|=|A-A|+1$.

Proof. One may verify that $B$ is symmetric, that $A-A=B-B$ and that $A+A=(B+B) \sqcup\{2 a\}$.

Remark. The set $A_{8}$ is the case $n=d=2$ of this theorem.

Next we turn to $A_{6}$. Note that $A_{6}=A_{1} \cup\{16\}$ and that it does not have a symmetric subset of size 8 . The following generalises $A_{6}$ in a different direction to the generalisation of $A_{1}$ given in [4]: 
Theorem 5. Let $n \geq 2, k \geq 3$. Set

$$
\begin{aligned}
X:= & \{2 j: 0 \leq j<n\}, \quad m:=2(k+1) n-2, \quad Y:=m-X, \\
Z:= & \{2 j n-1: 1 \leq j \leq k\}, \quad B:=X \sqcup Y \sqcup Z, \quad a^{*}:=2 n, \\
& A^{*}:=B \sqcup\left\{a^{*}\right\}, \quad a:=m+2, \quad A:=A^{*} \sqcup\{a\} .
\end{aligned}
$$

Then $A^{*}$ and $A$ are both MSTD sets and $\left|A^{*}+A^{*}\right|-\left|A^{*}-A^{*}\right|=|A+A|-$ $|A-A|=1$.

REMARK. The sets $A$ in Theorem 5 provide explicit examples of MSTD sets which are not obtained from a symmetric subset by adding a single element, followed possibly by a base expansion. Indeed, $B$ is a maximal symmetric subset of $A$ and $|A \backslash B|=2$. Finally, note that the set $A_{6}$ is the case $n=2, k=3$ of the theorem.

Proof of Theorem 5. One may verify the following facts, from which the theorem follows:

(i) $B$ is symmetric.

(ii) $A^{*}-A^{*}=B-B$.

(iii) $A^{*}+A^{*}=(B+B) \sqcup\left\{2 a^{*}\right\}$.

(iv) $A-A=\left(A^{*}-A^{*}\right) \sqcup\left\{ \pm a, \pm\left[a-\left(a^{*}-1\right)\right]\right\}$.

(v) $A+A=\left(A^{*}+A^{*}\right) \sqcup\left\{2 a, 2 a-2, a+(2 k n-1), a+a^{*}\right\}$.

Our fourth construction is a novel generalisation of the set $A_{4}$, different from that covered by Theorem 1 of [4]:

Theorem 6. Let $n, k>1$. Set

$$
\begin{aligned}
& X:=[0, n], \quad m:=(2 k+3) n, \quad Y:=m-X, \\
& Z:=\bigsqcup_{j=1}^{k}[2 j n+1,(2 j+1) n-1], \\
& B:=X \sqcup Y \sqcup Z, \quad a:=2 n, \quad A:=B \sqcup\{a\} .
\end{aligned}
$$

Then $A$ is an MSTD set with $|A+A|-|A-A|=1$. Furthermore, set

$$
W:=[(2 k+4) n,(2 k+5) n), \quad \mathcal{A}:=A \sqcup W .
$$

Then $\mathcal{A}$ is also an $M S T D$ set and $|\mathcal{A}+\mathcal{A}|-|\mathcal{A}-\mathcal{A}|=2$.

Proof. One may verify the following facts, from which the theorem follows:

(i) $B$ is symmetric.

(ii) $A-A=B-B$.

(iii) $A+A=(B+B) \sqcup\{2 a\}$.

(iv) $\mathcal{A}-\mathcal{A}=(A-A) \sqcup \pm[(2 k+3) n+1,(2 k+5) n-1]$. Hence $|\mathcal{A}-\mathcal{A}|=$ $|A-A|+(4 n-2)$. 
(v) $\mathcal{A}+\mathcal{A}=(A+A) \sqcup[(4 k+6) n+1,(4 k+10) n-2]$. Hence $|\mathcal{A}+\mathcal{A}|=$
$|A+A|+(4 n-1)$.

Remarks. (a) In the case $n=k=2$, the set $A$ coincides with $A_{4}$ and the set $\mathcal{A}$ is $A_{4} \cup\{16,17\}=\{0,1,2,4,5,9,12,13,14,16,17\}$. Denote this set by $A_{12}$ for future reference.

(b) The sets $\mathcal{A}$ provide an explicit infinite family of examples of MSTD sets in which the size of the sumset is at least two more than the size of the difference set, and which are not obtained from a fixed MSTD set by either a base expansion or by the method of Lemma 7 below (the latter method is implicit in [4]).

(c) The set $A_{2}$ has as a superset $A_{13}=A_{2} \cup\{18,19,20\}$ which also satisfies $\left|A_{13}+A_{13}\right|-\left|A_{13}-A_{13}\right|=2$. This set is not, however, covered by Theorem 6 , and we have not spotted a way to generalise either $A_{13}$ or $A_{2}$ itself.

(d) Given an MSTD set $M$, let $s(M)$ denote the maximal size of a symmetric subset of $M$. In Theorem 5 , we gave examples in which $|M|-s(M)$ $=2$. All our examples prior to that satisfy $|M|-s(M)=1$.

Now consider the sets $\mathcal{A}$ in Theorem 6 . Each $\mathcal{A}$ has the symmetric subset $B$ and $|\mathcal{A} \backslash B|=n+1$. Let $C=\{n,(2 k+3) n\} \sqcup(Z \backslash[2 n+1,3 n-1])$. Then $\mathcal{A} \backslash C$ is also symmetric and $|\mathcal{A} \backslash C|=(k-1)(n-1)+2$. It is easily checked that $\mathcal{A}$ has no symmetric subset which is larger than both $B$ and $\mathcal{A} \backslash C$. Thus we get examples of MSTD sets $M$ in which the difference $|M|-s(M)$ can be made arbitrarily large. The sets constructed in the proof of Theorem 8 below provide further examples of this phenomenon. Again, the point is that in both cases, these examples are not base expansions of a fixed set.

4. How much larger can the sumset be? Starting from any fixed MSTD set, the base expansion method allows one to construct MSTD sets $A$ for which the quotients $|A+A| /|A-A|$ become arbitrarily large. Here we are interested in both the quotient $|A+A| /|A-A|$ and the difference $|A+A|-|A-A|$. Our first step is to describe a way to identify an MSTD subset $A$ of $\mathbb{Z}$ with MSTD subsets of $\mathbb{Z} / n \mathbb{Z}$ for suitable $n$ depending on $A$. This idea is also implicitly contained in [4].

Let $A$ be a normalised MSTD subset of $\mathbb{Z}$, and $m:=\max \{a \in A\}$. Then $A$ can be considered as an MSTD subset of $\mathbb{Z} / n \mathbb{Z}$ for any $n>2 m$. More generally, we make the following definition:

Definition. Let $A$ be a normalised subset of $\mathbb{Z}$ (not necessarily an MSTD set) with largest element $m$, and $n>0$ an integer. Set $A^{\prime}:=A \cap[0, n)$ and identify $A^{\prime}$ with a subset of $\mathbb{Z} / n \mathbb{Z}$ in the natural way. We call $A^{\prime}$ the reduction of $A$ modulo $n$. Then $A$ is said to be reducible modulo $n$ if $A=$ 
$\left\{x \in[0, m]: x(\bmod n) \in A^{\prime}\right\}$. If, in addition, $A^{\prime}$ is an MSTD set in $\mathbb{Z} / n \mathbb{Z}$, we say that $A$ has good MSTD-reduction modulo $n$.

An important observation, which is basically a reformulation and sharpening of Theorem 8 of [4] in the case of cyclic groups, is the following:

Lemma 7. Let $A$ be a normalised subset of $\mathbb{Z}$ and $n>0$ an integer. Suppose $A$ is reducible modulo $n$ with reduction $A^{\prime}$. Let

$$
f(n, A):=\left|A^{\prime}+A^{\prime}\right|-\left|A^{\prime}-A^{\prime}\right| .
$$

Put $B:=A \cup(A+n)$. Then $B$ is a normalised subset of $\mathbb{Z}$ and

$$
|B+B|-|B-B|=(|A+A|-|A-A|)+2 \cdot f(n, A) .
$$

Proof. Let $X$ be any congruence class of integers modulo $n$. The reducibility of $A$ modulo $n$ implies the following. If $x_{1}, x_{2} \in X \cap A+A$ (resp. $X \cap A-A$ ), and $x_{3} \in\left(x_{1}, x_{2}\right) \cap X$, then $x_{3} \in X \cap A+A$ (resp. $X \cap A-A$ ). Furthermore, $X \cap A+A$ (resp. $X \cap A-A$ ) is non-empty if and only if $X \cap B+B$ (resp. $X \cap B-B$ ) is.

Suppose $X \cap A+A$ is non-empty with largest element $x$. It follows that $X \cap B+B=(X \cap A+A) \sqcup\{x+n, x+2 n\}$. Similarly, suppose $X \cap A-A$ is nonempty with largest element $x_{0}$ and smallest element $x_{1}$. Then $X \cap B-B=$ $(X \cap A-A) \sqcup\left\{x_{0}+n, x_{1}-n\right\}$.

(24) follows immediately from these observations.

Each of the sets listed on page 66 has good MSTD reduction modulo $n$, for some $n$ considerably less than $2 \cdot \max \{a \in A\}$. For example, we can take

$$
\begin{aligned}
& A_{4}^{\prime}=A_{4} \backslash\{12,13,14\}, n=12, \quad f\left(12, A_{4}\right)=1, \\
& A_{2}^{\prime}=A_{2}, \quad n=18, \quad f\left(18, A_{2}\right)=1 \text {, } \\
& A_{5}^{\prime}=A_{5} \backslash\{16\}, \quad n=16, \quad f\left(16, A_{5}\right)=1 \text {, } \\
& A_{6}^{\prime}=A_{6}, \quad n=21, \quad f\left(21, A_{6}\right)=2 \text {, } \\
& A_{7}^{\prime}=A_{7} \backslash\{16,18\}, \quad n=16, \quad f\left(16, A_{7}\right)=1, \\
& A_{8}^{\prime}=A_{8} \backslash\{17,19\}, \quad n=17, \quad f\left(17, A_{8}\right)=1 \text {, } \\
& A_{9}^{\prime}=A_{9} \backslash\{17,21\}, \quad n=17, \quad f\left(17, A_{9}\right)=1 \text {, } \\
& A_{10}^{\prime}=A_{10} \backslash\{17,22\}, \quad n=17, \quad f\left(17, A_{10}\right)=1 \text {, } \\
& A_{11}^{\prime}=A_{11} \backslash\{21,25\}, \quad n=21, \quad f\left(21, A_{11}\right)=1 .
\end{aligned}
$$

More generally, in the notation of the theorems above:

- Theorem 2: For given $n, k, d$ the set $A$ has good MSTD reduction modulo $r:=\left(2^{n+1}+d\right)+\left(2^{n-1}-2^{k}\right)$ and $f(r, A)=1$.

- Theorem 3: For given $n, l$ the set $A$ has good MSTD reduction modulo $r:=\left(2^{n+1}-1\right)+\left(2^{n-1}-2^{l}\right)$ and $f(r, A)=1$.

- Theorem 4: For given $n, d$ the set $A$ has good MSTD reduction modulo $r:=4 n d+1$ and $f(r, A)=1$. 
- Theorem 5: For given $n, k$ the set $A$ has good MSTD reduction modulo $r:=2(k+2) n+1$. If $k=3$ then $f(r, A)=2$. If $k>3$ then $f(r, A)=1$.

- Theorem 6: For given $n, k$ the set $A$ has good MSTD reduction modulo $r:=(2 k+2) n$ and $f(r, A)=1$. Note that, in fact, $A+A(\bmod r)=$ $\mathbb{Z} / r \mathbb{Z}$ and $A-A(\bmod r)=\mathbb{Z} / r \mathbb{Z} \backslash\{r / 2\}$.

The rather tedious proofs of these statements are omitted. Instead we note that of particular interest is the fact that $f\left(21, A_{6}\right)>1$. This means that when repeatedly applying Lemma 7 , the difference in size between the sum- and difference sets will grow more quickly. Let

$$
\begin{aligned}
A_{14}:= & A_{6} \sqcup\left(A_{6}+21\right) \sqcup\left(A_{6}+42\right) \\
= & \{0,2,4,5,9,12,13,14,16,21,23,25,26,30, \\
& 33,34,35,37,42,44,46,47,51,54,55,56,58\} .
\end{aligned}
$$

Then $\left|A_{14}+A_{14}\right|=114$ and $\left|A_{14}-A_{14}\right|=105$, so in the notation of (1), we have $f\left(A_{14}\right)=\ln 114 / \ln 105=1.01767 \ldots$ Thus $f\left(A_{14}\right)>f\left(A_{3}\right)$. By the way, note that $A_{3}=A_{12} \cup\left(A_{12}+12\right)$.

The following example does even better: Let

$$
X:=\{0,1,2,4,5,9,12,13,17,20,21,22,24,25\}
$$

(this set appears in [3], where it is denoted $S_{4}$ ). Then $|X+X|-|X-X|=4$, $X$ is reducible modulo 20 and $f(20, X)=2$. Take

$A_{15}:=X \cup(X+20)$

$=\{0,1,2,4,5,9,12,13,17,20,21,22,24,25,29,32,33,37,40,41,42,44,45\}$.

Then $\left|A_{15}+A_{15}\right|=91$ and $\left|A_{15}-A_{15}\right|=83$, so that $f\left(A_{15}\right)=\ln 91 / \ln 83=$ $1.02082 \ldots$

Our final result resolves in the affirmative Conjecture 20 of [3]:

THEOREM 8. Let $j, k$ be any two non-negative integers. Then there exists a constant $c_{j, k} \in(0,1)$ such that, for all $n$ sufficiently large, depending on $j$ and $k$, at least $c_{j, k} \cdot 2^{n+1}$ of the subsets $A$ of $\{0,1, \ldots, n\}$ satisfy $|A+A|=(2 n+1)-j$ and $|A-A|=(2 n+1)-2 k$.

Proof. The proof is an extension of the method of [3] and consists of two separate constructions depending on the sign of $j-k$.

Case $I: j \leq k$. We make use of the set $A_{12}=\{0,1,2,4,5,9,12,13,14$, $16,17\}$ above. It satisfies $A_{12}+A_{12}=[0,34]$ and $A_{12}-A_{12}=[-17,17] \backslash\{ \pm 6\}$. Furthermore, this set has good MSTD reduction modulo 12 .

Let $j, k$ be given. Set

$$
X_{k}:=\bigcup_{t=0}^{k-1} A_{12}+12 t .
$$


Let $m_{k}=\max X_{k}=12 k+5$. By the proof of Lemma 7 , we have $X_{k}+X_{k}=$ $\left[0,2 m_{k}\right]$ and $\left|X_{k}-X_{k}\right|=\left(2 m_{k}+1\right)-2 k$, since the difference set misses all numbers congruent to $6(\bmod 12)$ in the interval $\left[-m_{k}, m_{k}\right]$. Now set

$$
X_{j, k}:=X_{k} \backslash\{12(k-t)+1: 0 \leq t<j\} .
$$

One readily checks that $X_{j, k}-X_{j, k}=X_{k}-X_{k}$ but $X_{j, k}+X_{j, k}=[0,2 m] \backslash$ $\{24(k-t)+3: 0 \leq t<j\}$. Thus the sets $X_{j, k}$ already provide explicit examples of sets satisfying the requirements of Theorem 8 . To prove the existence of positive constants $c_{j, k}$ we use the method of [3]. Set $L_{j, k}:=$ $\left(m_{k}-X_{j, k}\right) \sqcup\left(m_{k}, 2 m_{k}\right]$ and $U_{j, k}:=n-\left(X_{k} \sqcup\left(m_{k}, 2 m_{k}\right]\right)$, for any $n \geq 4 m_{k}+1$. Finally, put

$$
A_{j, k}:=L_{j, k} \sqcup R \sqcup U_{j, k},
$$

where $R$ is the random subset of $\left(2 m_{k}, n-2 m_{k}\right)$ obtained by choosing each number in the interval independently with probability $1 / 2$. Our choices of $L_{j, k}$ and $U_{j, k}$ imply that

$$
\begin{aligned}
\#\left\{\left(\left[0,4 m_{k}\right] \cup\left[2 n-4 m_{k}, 2 n\right]\right) \backslash\left(A_{j, k}+A_{j, k}\right)\right\} & =j, \\
\#\left\{\left[n-4 m_{k}, n\right] \backslash\left(A_{j, k}-A_{j, k}\right)\right\} & =k .
\end{aligned}
$$

It then suffices to apply the same type of argument as in [3] to show that, with high probability, both $\left(4 m_{k}, 2 n-4 m_{k}\right) \subset A_{j, k}+A_{j, k}$ and $\left[0, n-4 m_{k}\right) \subset$ $A_{j, k}-A_{j, k}$, and thus deduce the existence of a constant $c_{j, k}>0$ as desired.

Case $I I: j \geq k$. We start by describing, for each $j \geq 0$, an integer $m_{j} \geq-1$ and a subset $L_{j} \subset\left[0, m_{j}\right]$ with the following properties:

(i) $L_{j}+L_{j}=\left[0,2 m_{j}\right] \backslash\left\{2 x_{1}, \ldots, 2 x_{j}\right\}$ where $0<x_{1}<\cdots<x_{j}<m_{j} / 2$.

(ii) None of the numbers $2 x_{u}-x_{v}$, for $1 \leq u, v \leq j$, is in $L_{j}$.

Set $m_{0}:=-1, L_{0}:=\emptyset$. This is consistent with (i) and (ii) above. We define the numbers $m_{1}<m_{2}<\cdots$ and the sets $L_{1} \subset L_{2} \subset \cdots$ inductively as follows: for each $i \geq 0$,

$$
\begin{aligned}
m_{i+1} & :=3 m_{i}+16, \\
L_{i+1} & :=L_{i} \sqcup\left\{\left(m_{i}+1\right)+\left\{0,1,2,5, m_{i}+10, m_{i}+11, \ldots, 2 m_{i}+15\right\}\right\} .
\end{aligned}
$$

Each $m_{j}$ is an odd number and one readily checks that

$$
\begin{aligned}
m_{j} & =7 \cdot 3^{j}-8, \\
\left|L_{j}\right| & =\frac{7}{2}\left(3^{j}-1\right)+2 j, \\
L_{j}+L_{j} & =\left[0,2 m_{j}\right] \backslash\left\{2 x_{t}=2\left(m_{t}+5\right): 0 \leq t<j\right\} .
\end{aligned}
$$

It is then a simple exercise to show that conditions (i) and (ii) above on $L_{j}$ are satisfied.

Now let $j, k$ be given with $j \geq k$ and first suppose $k>0$. Set

$$
L_{j, k}:=L_{j} \sqcup\left\{2 x_{t+1}-x_{t}: 1 \leq t \leq j-k\right\},
$$


and observe that the numbers $x_{t}$ grow sufficiently fast so that for any $k$, $L_{j, k}+L_{j, k}=L_{j}+L_{j}$. For any $n \geq 2 m_{j}+1$ set

$$
U_{j, k}=U_{j}:=n-\left(L_{j} \cup\left\{x_{1}, \ldots, x_{j}\right\}\right) .
$$

Then, as usual, take finally $A_{j, k}:=L_{j, k} \sqcup R \sqcup U_{j, k}$, where $R$ is the random subset of $\left(m_{j}, n-m_{j}\right)$ obtained by choosing each number independently with probability $1 / 2$. One easily checks that

$$
\begin{gathered}
\left(\left[0,2 m_{j}\right] \cup\left[2 n-2 m_{j}, 2 n\right]\right) \backslash\left(A_{j, k}+A_{j, k}\right)=\left\{2 x_{t}: t=1, \ldots, j\right\}, \\
{\left[n-2 m_{j}, n\right] \backslash\left(A_{j, k}-A_{j, k}\right)=\left\{n-1-2 x_{t}: t=1 \text { or } j-k+2 \leq t \leq j\right\} .}
\end{gathered}
$$

By the method of [3] both $\left(2 m_{j}, 2 n-2 m_{j}\right) \subset A_{j, k}+A_{j, k}$ and $\left[0, n-2 m_{j}\right)$ $\subset A_{j, k}-A_{j, k}$ occur with high probability, from which we deduce the existence of a positive constant $c_{j, k}$.

When $k=0$ we just have to be a little careful. One may check that the following choices work:

$$
L_{j, 0}:=L_{j+2} \sqcup\left\{x_{1}\right\} \sqcup\left\{2 x_{t+1}-x_{t}: 1 \leq t \leq j+1\right\}, \quad U_{j, 0}:=U_{j+2},
$$

and thus the proof of Theorem 8 is complete.

REMARKS. (a) In each of the above constructions we get explicit examples of sets satisfying the requirements of Theorem 8 by taking the set $R$ to consist of the entire interval over which it is defined. These examples are not covered by any of the constructions in Section 3, and in particular that in Case II is somewhat different from any of the earlier ones.

(b) From the above proof we obtain estimates of the form $c_{j, k}=$ $\Omega\left(2^{-\Theta\left(m_{l}\right)}\right)$, where $l=\max \{j, k\}$. The numbers $m_{l}$ grow linearly when $j<k$ but exponentially when $k>j$. This lack of symmetry is unsatisfying, though in any case the estimates are likely to be way smaller than the truth for all values of $j$ and $k$.

5. Concluding remarks. We have provided various explicit constructions of non-trivial infinite families of MSTD sets. All our constructions, including those in the proof of Theorem 8, and in common with those already in the literature, are roughly based on some type of symmetric set which is "perturbed" slightly by adding on a small number of elements. Often, though not always, the symmetric set is itself constructed from some (generalised) arithmetic progression. Theorems 2 and 3 provide an example where this is not so: there is an arithmetic progression $Z$ in the former construction, but both are based on a geometric progression $X$. The sets constructed in the proof of Theorem 8 are also of a somewhat different character. Note that in Theorem 6 , the set $Z$ is a GAP of dimension 2. We have not investigated whether the arithmetic progressions appearing in our various constructions can be replaced, as in [4], by GAPs of higher di- 
mension. More interesting, though, would be to have explicit examples of MSTD sets which are, in some meaningful sense, "radically" different from the blueprint of a perturbed symmetric set. The ubiquity of MSTD sets, as exhibited by probabilistic techniques to which Theorem 8 is our contribution, mean that such examples should/must exist. Note, by the way, that we have been unable to provide non-trivial families of MSTD sets which generalise the sets $A_{2}$ and $A_{10}$.

Otherwise, an outstanding open problem seems to be to obtain a reasonably tight estimate for $L:=\limsup _{|A| \rightarrow \infty} f(A)$, where $f$ is the function of (1). The set $A_{15}$ leaves the yawning gap $\ln 91 / \ln 83 \leq L \leq 4 / 3$. Finding good estimates for the constants $c_{j, k}$ in Theorem 8 is also a very appealing problem and several questions in a similar vein are suggested by the material in [3].

Finally, one would obviously like to be able to complete the classification, up to linear transformation, of MSTD sets of size 9, and if possible extend the range of computation to larger sizes. An interesting question is to find the smallest $n_{0}>0$ such that there are infinitely many different normalised MSTD sets of size $n_{0}$. All our constructions, plus those of [4], provide only finitely many sets of any given size. An upper bound is $n_{0}=16$, for which we can argue as follows: If $A, B \subset \mathbb{Z}$ and $C=A \times B \subset \mathbb{Z}^{2}$, then we have $|C \pm C|=|A \pm A| \cdot|B \pm B|$. In particular, if $A$ is an MSTD set and $B$ has at least as many sums as differences, then $C$ is an MSTD set in $\mathbb{Z}^{2}$. For suitable $\lambda, \mu \in \mathbb{N}$ the map $(a, b) \mapsto \lambda a+\mu b$ takes $C$ to a set $C_{\lambda, \mu} \subset \mathbb{Z}$ for which $\left|C_{\lambda, \mu} \pm C_{\lambda, \mu}\right|=|C \pm C|$ - this is just the base expansion method. If $\min \{|A|,|B|\}>1$ then there is a linear transformation taking $C_{\lambda_{1}, \mu_{1}}$ to $C_{\lambda_{2}, \mu_{2}}$ if and only if $\left|\begin{array}{ll}\lambda_{1} & \mu_{1} \\ \lambda_{2} & \mu_{2}\end{array}\right|=0$. In particular, we get infinitely many different sets up to linear transformation, each of size $|A||B|$. Since there is an MSTD set of size 8 , namely $A_{1}$, and we may take $B=\{0,1\}$ so that $|B+B|=|B-B|=3$, we may conclude that there are infinitely many normalised MSTD sets in $\mathbb{Z}$ of size 16 .

Acknowledgements. I thank Friedrich Roesler for his observations which led to some of the discoveries in this paper.

\section{References}

[1] G. A. Freiman and V. P. Pigarev, The relation between the invariants $R$ and $T$, in: Number-Theoretic Studies in the Markov Spectrum and in the Structural Theory of Set Addition, Kalinin. Gos. Univ., Moscow, 1973, 172-174 (in Russian).

[2] J. Marica, On a conjecture of Conway, Canad. Math. Bull. 12 (1969), 233-234.

[3] G. Martin and K. O'Bryant, Many sets have more sums than differences, in: Proceedings of CRM-Clay Conference on Additive Combinatorics (Montréal, 2006), to appear. 
[4] M. B. Nathanson, Sets with more sums than differences, Integers 7 (2007), A5, $24 \mathrm{pp}$.

[5] -, Problems in additive number theory, I, in: Proceedings of CRM-Clay Conference on Additive Combinatorics (Montréal, 2006), to appear.

[6] F. Roesler, A mean value density theorem of additive number theory, Acta Arith. 96 (2000), 121-138.

[7] I. Z. Ruzsa, On the cardinality of $A+A$ and $A-A$, in: Combinatorics (Keszthely, 1976), Colloq. Math. Soc. János Bolyai 18, Akadémiai Kiadó, Budapest, 1979, 933-938.

[8] —, Sets of sums and differences, in: Séminaire de Théorie des Nombres, Paris 1982/83, Birkhäuser, 1984, 267-273.

[9] -, An application of graph theory to additive number theory, Scientia 3 (1991), 97-109.

[10] -, On the number of sums and differences, Acta Math. Hungar. 59 (1992), 439-447.

Department of Mathematical Sciences

Division of Mathematics

Chalmers University of Technology and Göteborg University

41296 Göteborg, Sweden

E-mail: hegarty@math.chalmers.se

Received on 12.1.2007

and in revised form on 8.7.2007 